\title{
Extraction of maize vegetation coverage based on UAV multi-spectral remote sensing and pixel dichotomy
}

\author{
Dongjian Yang ${ }^{1,2}$, Yubin Lan ${ }^{2,3,4,5}$, Wenhua Li ${ }^{1,2}$, Chuanxu Hu ${ }^{1,2}$, \\ Haiyu Xu ${ }^{1,2}$, Jianchi Miao ${ }^{1,2}$, Xiao Xiao ${ }^{1,2}$, Lianbin $\mathrm{Hu}^{1,2}$, Daocai Gong ${ }^{1,2}$, Jing Zhao ${ }^{1,2^{*}}$ \\ (1. Shandong University of Technology, School of Agricultural Engineering and Food Science, Zibo 255000, Shandong, China; \\ 2. National Sub-center for International Collaboration Research on Precision Agricultural Aviation Pesticide Spraying Technology, \\ Shandong University of Technology Sub-center of National Center for International Collaboration Research on Precision Agricultural \\ Aviation Pesticide Spraying Technology, Zibo 255000, China; \\ 3. National Center for International Collaboration Research on Precision Agricultural Aviation Pesticides Spraying Technology/ \\ College of Engineering, South China Agricultural University, Guangzhou 510642, China; \\ 4. Department of Biological and Agricultural Engineering, Texas A\&M University, College Station, Texas, 77843, USA; \\ 5. Texas A\&M AgriLife Research and Extension Center, Beaumont, Texas, 77713, USA)
}

\begin{abstract}
In order to quickly and accurately obtain the vegetation coverage information at the jointing stage of summer maize, drone remote sensing was used to obtain the multi-spectral images of unmanned farms. Seven multi-spectral vegetation indices were extracted from the images, including normalized vegetation index (NDVI), normalized green belt difference vegetation index (GNDVI)), enhanced vegetation index (EVI), difference vegetation index (DVI), soil adjusted vegetation index (SAVI), ratio vegetation index (RVI), and optimal soil adjusted vegetation index (OSAVI) vegetation coverage extraction model. Using Support Vector Machine supervised classification method was adopted to obtain the true value of vegetation coverage of summer maize jointing period and the calculation accuracy of each vegetation coverage extraction model was evaluated. In order to ensure the accuracy and stability of the extraction results, 50 independent verification units were selected in the experimental areas, and the true value of the vegetation coverage in each area was linearly fitted with the vegetation coverage predicted value obtained by the vegetation coverage extraction model, and each was calculated. The coefficient of determination $\left(R^{2}\right)$ and the root mean square error (RMSE) of the vegetation coverage extraction model, were compared with the fitting accuracy and goodness of fit of the linear regression models, and finally an optimal summer maize jointing stage coverage extraction model was obtained. The results show that the vegetation coverage extraction model constructed based on the pixel dichotomy combined with the multi-spectral vegetation index EVI has the best effect on the extraction of summer maize jointing stage coverage and the highest accuracy. Compared with the true value of vegetation coverage, the extraction error of the EVI vegetation coverage extraction model $\left(E_{F}\right)$ is $11.76 \%$ with $R^{2}$ of 0.9418 and RMSE of 0.0537. Based on the pixel dichotomy and using the multi-spectral vegetation index a vegetation coverage model was constructed, and the vegetation coverage of summer maize at the jointing stage can be quickly and accurately extracted. This study can provide technical support for the realization of precision agriculture.
\end{abstract}

Keywords: Unmanned farm, UAV, multispectral remote sensing image, pixel dichotomy, vegetation index, maize vegetation coverage

DOI: $10.33440 /$ j.ijpaa.20210402.172

Citation: Yang D J, Lan Y B, Li W H, Hu C X, Xu H Y, Miao J C, Xiao X, Hu L B, Gong D C, Zhao J. Extraction of maize vegetation coverage based on UAV multi-spectral remote sensing and pixel dichotomy. Int J Precis Agric Aviat,2021;4(2): 1-7.

\section{Introduction}

Maize has the status of "king of feed" in economic benefits and is an important food crop in China ${ }^{[1]}$. Nowadays, more and more

Received date: 2021-09-05 Accepted date:2021-10-20

Biographies: Dongjian Yang, Postgraduate, research interests: remote sensing, mechanical design and computer programming. Email: 694415363@qq.com; Yubin Lan, PhD, research interests: precision agricultural aviation application. Email: ylan@scau.edu.cn; Wenhua Li, Postgraduate, research interests: remote sensing. Email: 1252559859@qq.com; Chuanxu Hu, Postgraduate, research interests: remote sensing. Email: 416458843@qq.com; Haiyu Xu, Postgraduate, research interests: remote sensing. Email: 903837514@qq.co; Jianchi Miao, Postgraduate, research interests: Unmanned aerial remote sensing. Email: 1393746086@qq.com; Xiao Xiao, Postgraduate, research interests: Unmanned aerial remote sensing. Email: 1085751745@qq.com; Lianbin Hu, Postgraduate, research interests: remote sensing. Email: 1906800883@qq.com; Daocai Gong, Postgraduate, research interests: remote sensing. Email: 1252128352@qq.com; *Corresponding author: Jing Zhao, Associate professor, research interests: remote sensing, Mailing Address: Shandong University of Technology, Email: zbceozj@163.com. people pay attention to the role and value of maize. In order to ensure the stable development of the maize grain industry, it is necessary to pay more attention to the function and value of maize. The relevant physiological information is effectively monitored ${ }^{[2-3]}$ Vegetation coverage $^{[4]}$ (fractional vegetation cover, FVC) usually refers to the percentage of the total area of vegetation (stems, leaves, branches) projected vertically on the ground to the total area of the study area, which can characterize the photosynthesis capacity of green vegetation and growth conditions are important crop growth parameters. The monitoring of vegetation coverage mainly includes surface measurement method ${ }^{[5]}$ and remote sensing monitoring method. The surface measurement method requires a lot of manpower and material resources and has problems such as low efficiency. It is not suitable for large-area coverage evaluation. The remote sensing monitoring method uses UAVs. Or satellite remote sensing technology monitors the coverage of green areas, but satellite remote sensing is easily affected by the 
atmosphere, weather, etc., and satellite remote sensing data has low image resolution. It is often difficult to achieve high-precision crop field-scale coverage extraction. UAV remote sensing has the advantages of convenience and flexibility, few affected factors, timeliness and high resolution provide the possibility to accurately and efficiently extract the vegetation coverage at the crop field level. Based on TM remote sensing data, Li Miaomiao et $\mathrm{al}^{[6]}$ used pixel dichotomy to improve model parameters, and constructed a multi-spectral vegetation index (NDVI) vegetation coverage extraction model to achieve the extraction of vegetation coverage in the upper reaches of Miyun Reservoir with high accuracy. Zhao Jing et al. ${ }^{[7]}$ used UAV remote sensing technology to obtain visible light images of summer maize, extracted the three vegetation indices commonly used in visible light, VDVI, EXG and NGBDI, and used the method of combining supervised classification and vegetation index statistical histogram to use the threshold extraction method to achieve the coverage of summer maize at the field scale is quickly extracted. Niu Yaxiao et $\mathrm{al}^{[8]}$ independently developed a set of UAV multi-spectral image acquisition system based on open source flight control to obtain image information of four periods of winter wheat, and proposed a method of combining supervised classification and vegetation index statistical histogram. Scale extraction method of wheat coverage. The method of obtaining vegetation coverage through remote sensing has the advantages of fast and convenient, and the method of using satellite remote sensing technology to obtain vegetation coverage in the planting area has disadvantages such as high cost and poor timeliness. Using UAV remote sensing technology can efficiently and accurately obtain field-scale data. Compared with satellite remote sensing, vegetation coverage information has the advantages of high timeliness and low cost. Compared with visible light, multi-spectral images have more spectral information and have the advantages of efficiently and accurately extracting vegetation coverage. This paper uses UAV remote sensing technology to obtain multi-spectral images of unmanned farms, and uses pixel dichotomy to calculate 7 common multi-spectral vegetation indices to construct 7 vegetation coverage extraction models, and SVM (support vector machine) supervised classification is obtained. The result is used as the true value to evaluate the accuracy of the vegetation coverage extracted by each model, and finally obtain a grade map of the vegetation coverage of summer maize in the Jointing period, which provides technical support for realizing precision agriculture to guide field management.

\section{Materials and methods}

\subsection{Research area and data acquisition}

The test site is the ecological unmanned farm in Zhutai Town, Linzi District, Zibo City, Shandong Province (latitude $36^{\circ} 57^{\prime} 8^{\prime \prime}$, longitude $118^{\circ} 12^{\prime} 50^{\prime \prime}$ east). The area belongs to the temperate monsoon climate. The summer maize planting method is tractor sowing, the planting row spacing is $60 \mathrm{~cm}$, and the plant spacing is $20 \mathrm{~cm}$. The image data collection time is from 11:00-12:30 noon on July 20, 2020. The drone uses Shenzhen DJI Innovation Technology Co., Ltd. Jingwei M210V1 quad-rotor drone. The battery life is 20 minutes when fully charged. On the day of data collection, the weather was clear and there was no wind on the ground, which satisfies the flight requirements of the UAV platform. The machine is equipped with MS600Pro multi-spectral camera to obtain the orthophoto data of the test site. The image resolution is 1280 pixel $\times 960$ pixel, and the ground resolution is $0.88 \mathrm{~cm}$. The captured image is stored in the SD card. The experimental design UAV has a flying height of $50 \mathrm{~m}$, a flying speed of $4 \mathrm{~m} / \mathrm{s}$, a heading overlap of $85 \%$, and a lateral overlap of $85 \%$. A total of 2964 orthophotos of summer maize were obtained in the experiment. Further pre-processing the images, using pix4Dmapper software to perform geometric correction and stitching of the images. The research area after pretreatment is shown in Figure 1 below.

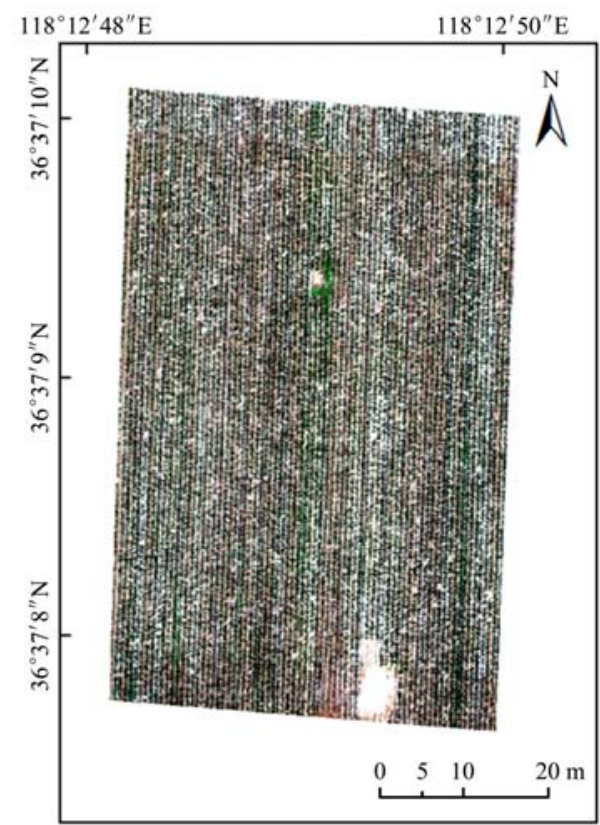

Figure 1 The experimental field

\subsection{Extraction method of summer maize vegetation coverage}

\subsubsection{Pixel dichotomy}

The commonly used remote sensing measurement methods for vegetation coverage estimation include: regression model $\operatorname{method}^{[9-14]}$, vegetation index method and pixel decomposition model method. The concept of pixel decomposition model method considers that a pixel in an image is composed of multiple components. The types of tables in each region all contribute to the pixel information, and the vegetation coverage can be estimated by using the pixel decomposition model.

In the pixel decomposition model method, the linear decomposition model is the most commonly used. In this paper, the simplest and most efficient pixel dichotomy method is selected to achieve efficient and accurate extraction of vegetation coverage.

The pixel dichotomy ${ }^{[15]}$ is as follows: suppose that a piece of pixel information on an image is composed of two types of surface pixel information in the study of vegetation coverage. All the information of the image is composed of a weighted linear combination of the area ratio of vegetation pixels and soil pixels, which can be obtained:

$$
S=F V C \times S_{\text {veg }}+(1-F V C) \times S_{\text {soil }}
$$

In the formula, $S$ is the spectral information of the pixel, FVC is the vegetation coverage, Vegetation coverage refers to the ratio of the vertical projection area of vegetation (including leaves, stems, branches) in a certain area to the total area of the area, expressed as a percentage. The formula for calculating vegetation coverage using the pixel dichotomy is:

$$
F V C=\left(S-S_{\text {soil }}\right) /\left(S_{\text {veg }}-S_{\text {soil }}\right)
$$

In the formula, $S_{\text {soil }}$ and $S_{\text {veg }}$ represent the soil pixel information and vegetation pixel information in the pixel, respectively.

\subsubsection{Construction of vegetation coverage extraction model}

According to the spectral characteristics of vegetation, the 
spectral bands collected by the sensor are combined and calculated to form various vegetation indices. The vegetation indices can be used to simply and efficiently measure the surface vegetation conditions, which is an empirical and powerful vegetation observation.

Because green vegetation has strong absorption of red light and high reflection and high transmission in the near infrared, healthy green vegetation has a large difference in reflectivity between NIR and $R$. It can be seen that the reflectivity of $\mathrm{R}$ and NIR is combined and the calculated vegetation index can reflect the growth of vegetation to a certain extent. In this paper, we choose to use multi-spectral data to have more spectral information than visible light images and to collect NIR reflectance. The 7 planting index commonly used in multi-spectrum is selected ${ }^{[16]}$. Normalized difference vegetation index (NDVI), normalized difference vegetation index (GNDVI), enhanced vegetation index (EVI), difference Vegetation index (Difference vegetation index, DVI), soil adjusted vegetation index (Soil adjusted vegetation index. SAVI), ratio vegetation index (Ratio vegetation index, RVI) and optimal soil adjusted vegetation index (Optimized soil adjusted vegetation index, OSAVI) for the construction of vegetation coverage model, the vegetation index and calculation formula are as follows:

Table 1 Vegetation index

\begin{tabular}{|c|c|c|}
\hline Vegetation index & Name & Calculation formula \\
\hline$N D V I$ & $\begin{array}{l}\text { Normalized Difference } \\
\text { Vegetation Index }\end{array}$ & $\left(R_{N i r}-R_{R e d}\right) /\left(R_{N i r}+R_{R e d}\right)$ \\
\hline GNDVI & $\begin{array}{c}\text { Green Normalized Difference } \\
\text { Vegetation Index }\end{array}$ & $\left(R_{\text {Nir }}-R_{\text {Green }}\right) /\left(R_{\text {Nir }}+R_{\text {Green }}\right)$ \\
\hline EVI & Enhanced Vegetation Index & $\begin{array}{c}2.5\left(R_{\text {Nir }}-R_{\text {Red }}\right) / \\
\left(R_{\text {Nir }}+6.0 R_{\text {Red }}-7.5 R_{\text {Blue }}+1\right)\end{array}$ \\
\hline$D V I$ & Difference Vegetation Index & $R_{N i r}-R_{R e d}$ \\
\hline SAVI & $\begin{array}{l}\text { Soil Adjustment Vegetation } \\
\text { Index }\end{array}$ & $1.5\left(R_{N i r}-R_{R e d}\right) /\left(R_{N i r}+R_{R e d}+0.5\right)$ \\
\hline$R V I$ & Ratio Vegetation Index & $R_{N i r} / R_{R e d}$ \\
\hline OSAVI & $\begin{array}{l}\text { Optimal Soil Adjustment } \\
\text { Vegetation Index }\end{array}$ & $\left(R_{N i r}-R_{R e d}\right) /\left(R_{N i r}+R_{R e d}+0.16\right)$ \\
\hline
\end{tabular}

In the table, $R_{N i r}$ is the reflectance in the near-infrared band (compared experimentally, the result of $840 \mathrm{~nm}$ in the two near-infrared bands of $840 \mathrm{~nm}$ and $940 \mathrm{~nm}$ is better than $940 \mathrm{~nm}$, so the selection of the near-infrared band in this article uses $840 \mathrm{~nm}$ for data processing), $R_{\text {Red }}$ is red light the reflectivity of the wave band, $R_{\text {Green }}$ is the reflectivity of the green light band, and $R_{\text {Blue }}$ is the reflectivity of the blue light band.

Multi-spectral vegetation index is obtained by the spectral information of the ground object collected by the sensor and calculated by the band, which can quantitatively reflect the value of the ground surface information. In the pixel dichotomy, the value of a pixel on the image is the sum of the soil pixel and the vegetation pixel. By the pixel dichotomy and formula (2):

$$
\begin{aligned}
& \mathrm{FVC}_{\mathrm{NDVI}}=\left(\mathrm{NDVI}-\mathrm{NDVI}_{\mathrm{soil}}\right) /\left(\mathrm{NDVI}_{\mathrm{veg}}-\mathrm{NDVI}_{\mathrm{soil}}\right) \\
& \mathrm{FVC}_{\mathrm{GNDVI}}=\left(\mathrm{GNDVI}-\mathrm{GNDVI}_{\mathrm{soil}}\right) /\left(\mathrm{GNDVI}_{\mathrm{veg}}-\mathrm{GNDVI}_{\mathrm{soil}}\right) \\
& \mathrm{FVC}_{\mathrm{EVI}}=\left(\mathrm{EVI}-\mathrm{EVI}_{\text {soil }}\right) /\left(\mathrm{EVI}_{\text {veg }}-\mathrm{EVI}_{\text {soil }}\right) \\
& \mathrm{FVC}_{\text {DVI }}=\left(\mathrm{DVI}-\mathrm{DVI}_{\text {soil }}\right) /\left(\mathrm{DVI}_{\text {veg }}-\mathrm{DVI}_{\text {soil }}\right) \\
& \mathrm{FVCSAVI}_{\text {Sa }}=\left(\mathrm{SAVI}-\mathrm{SAVI}_{\text {soil }}\right) /\left(\mathrm{SAVI} \mathrm{I}_{\text {veg }}-\mathrm{SAVI}_{\text {soil }}\right) \\
& \mathrm{FVC}_{\mathrm{RVI}}=\left(\mathrm{RVI}-\mathrm{RVI}_{\text {soil }}\right) /\left(\mathrm{RVI}_{\mathrm{veg}}-\mathrm{RVI}_{\text {soil }}\right)
\end{aligned}
$$

The FVC in the above formula is a formula based on the vegetation coverage extraction model established under the NDVI, NGDVI, EVI, DVI, SAVI, OSAVI vegetation index, which extracts the vegetation coverage. Thus, the extraction model of multi-spectral vegetation index coverage is obtained ${ }^{[17]}$. Based on the pixel dichotomy, the vegetation coverage can be extracted by using the vegetation index.

2.2.3 Extraction of summer maize vegetation coverage

The extraction process of summer maize vegetation coverage is shown in the Figure 2.

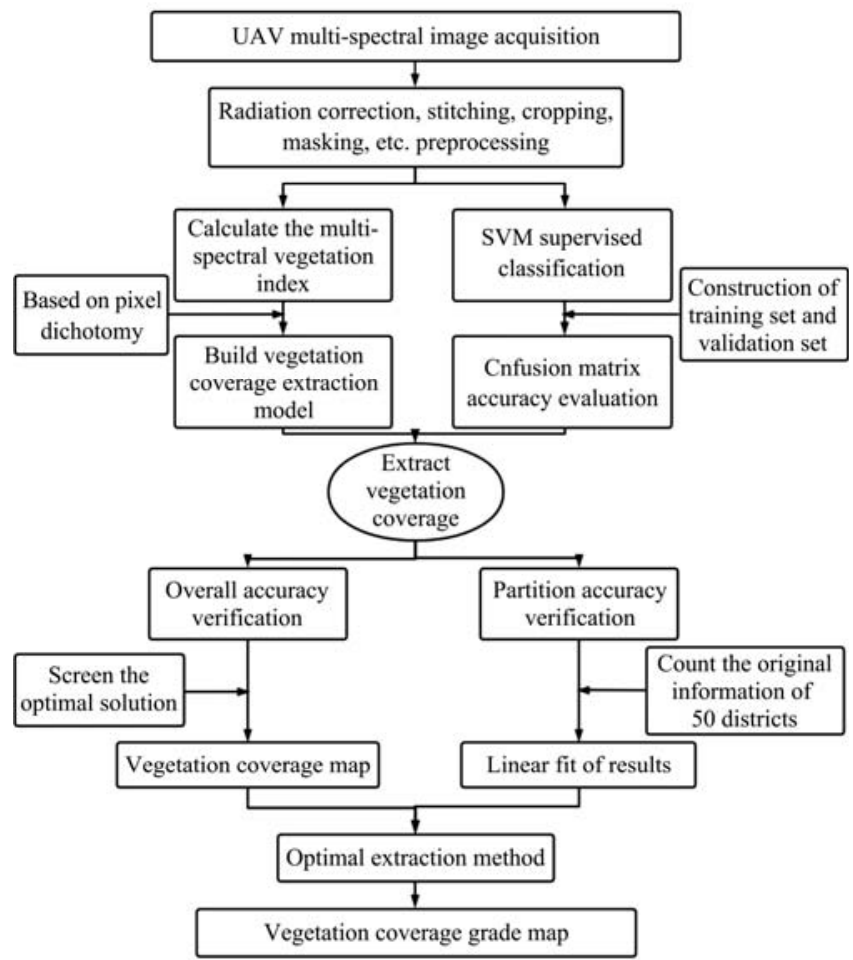

Figure 2 Data processing flow chart

The pre-processed image is based on the vegetation index calculation formula to obtain the 7 planting index map of the multispectral image. Referring to the pure pixel information estimation method of TM remote sensing image proposed by $\mathrm{Li}$ Miaomiao et al ${ }^{[6]}$, the values of $V I_{\text {soil }}$ and $V I_{\text {veg }}$ are as follows. The value of different images varies with time and space. Because $V I=V I_{\text {soil }}+V I_{\text {veg }}$, the value range of $V I_{\text {veg }}$ is also determined. This article uses UAV remote sensing technology to obtain multispectral image data of unmanned farms, which can be ignored for the influence of the atmosphere on $V I_{\text {soil }}$, a certain degree of confidence can be used to study the vegetation coverage of the field scale range $\mathrm{e}^{[18-25]}$. Based on the clarity of the obtained image ${ }^{[26]}$, through repeated comparisons, $2 \%$ confidence is taken as the pure pixel information of the bare land (that is, $V I_{\text {soil }}$ is $2 \%, V I_{\text {veg }}=$ $1-V I_{\text {soil }}=98 \%$ ), according to the formula (3) (9). The vegetation coverage extraction model and pure pixel information extract the vegetation coverage of each vegetation index map ${ }^{[27]}$.

The calculation of the true value of vegetation coverage adopts support vector machine (SVM) supervised classification combined with formula (10) to extract the vegetation coverage, and the preprocessed image is manually selected by means of visual interpretation. The training samples and verification samples of the land use the calculation results as the true values to verify the accuracy of the vegetation coverage obtained by the 7 vegetation index construction models.

$$
F V C_{\text {svm }}=\frac{N_{\text {maize }}}{N_{\text {maize }}+N_{\text {soil }}}
$$


Among them, $N_{\text {maize }}$ is the total number of maize pixel statistics, and $N_{\text {soil }}$ is the total number of naked land pixel statistics.

2.2.4 Accuracy evaluation of vegetation coverage

The overall accuracy evaluation plan for extracting vegetation coverage using the vegetation coverage extraction model is as follows: The calculation formula for the extraction error $E_{F}$ of vegetation coverage is as follows:

$$
E_{F}=\frac{\left|F_{\text {sup }}-F_{\mathrm{VI}}\right|}{F_{\text {sup }}} \times 100 \%
$$

Among them, $F_{\mathrm{VI}}$ is the summer maize coverage obtained using the pixel dichotomy and vegetation coverage model, and $F_{\text {sup }}$ is the summer maize coverage obtained using the SVM (support vector machine) supervised classification method.

To verify the accuracy of the above-mentioned model method for extracting the coverage of summer maize at the three-leaf stage, this paper designs a method of regional accuracy verification to verify the accuracy of the test area. In the test area, 50 accuracy verification plots are selected and the supervised classification results are extracted. The coverage information is taken as the true value, and the vegetation coverage results extracted from the 7 vegetation coverage models in the community are used as the predicted value. A linear regression model is established, and the root mean squared error (RMSE) and the coefficient of determination $\left(R^{2}\right)$ are used to compare Results are evaluated for accuracy. The smaller the RMSE, the closer the true value is to the predicted value; the greater the goodness of fit $R^{2}$ of the linear regression model, the better the fit between the true value and the predicted value.

\section{Results and analysis}

\subsection{Supervision classification results and evaluation}

By combining the two methods of field investigation and visual interpretation, 100 representative maize plant samples and 100 bare soil samples were selected on the multi-spectral image to form the training set, in order to ensure that different types of ground objects have good classification degree, the method of calculating JM (Jeffries-Matusita) distance ${ }^{[28,29]}$ is often used to calculate the separability of maize and bare land. Both parameter values are between 0 and 2.0. When the parameter value is greater than 1.9, the corresponding sample. The separability is good, if it is less than 1.8, the separability is poor and it is not suitable to continue the classification. At the same time, 60 locations of bare land and maize samples in the verification set were uniformly selected in the figure to calculate the confusion matrix of the classification results, and to verify the accuracy of the SVM supervision classification results.

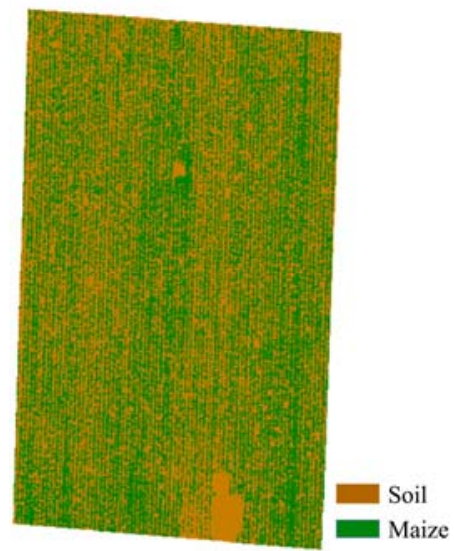

Figure 3 Summer maize supervised classification results
The separability of the two types of ground objects in the training set and the validation set selected for calculation is 1.999999989 and 1.99991650 , both of which are greater than 1.9, indicating that the two types of ground objects in the two sample sets have good separability. Further use the confusion matrix to pair. The classification accuracy is verified, and the overall classification accuracy is $99.4444 \%$, and the Kappa is 0.9889 . The detailed classification accuracy is shown in Table 2 .

Table 2 Evaluation of classification accuracy of summer maize

\begin{tabular}{lcccc}
\hline \multicolumn{1}{c}{ Category } & $\begin{array}{c}\text { Maize } \\
\text { pixel }\end{array}$ & $\begin{array}{c}\text { Soil } \\
\text { pixels }\end{array}$ & $\begin{array}{c}\text { Total sample } \\
\text { pixel }\end{array}$ & $\begin{array}{c}\text { User accuracy } \\
/ \%\end{array}$ \\
\hline Maize pixel & 23648 & 56 & 23704 & 99.776 \\
Soil pixels & 95 & 31143 & 31238 & 99.70 \\
Total sample pixel & 23743 & 31199 & 54942 & \\
User accuracy/\% & 99.60 & 99.82 & & \\
\hline
\end{tabular}

From Table 2, it can be seen that the use of SVM supervision classification to classify maize and bare land is better. Using formula (10) to calculate the average vegetation coverage of summer maize in the Jointing period is 0.443692, and the unmanned farm in the study area in summer. The vegetation coverage of maize in this period was $44.37 \%$. According to the research of previous scholars, this paper uses the SVM classification result as the true value to evaluate the accuracy of the model method to extract the vegetation coverage.

\subsection{Results and analysis of vegetation coverage extraction}

By counting the pixels of the multi-spectral vegetation index image, a total of $2 \%$ of the pixels are selected as the original information of the bare land and $98 \%$ are the original information of the maize. (In the process of image processing, the calculated value of the noise pixel value lower than the original information of the naked image is set to 0 by default, and the original pixel value of the noise image that is higher than the original information of the vegetation image is set to 1 ).

Statistic the $V I_{\text {soil }}$ and $V I_{\text {veg }}$ information of each VI (vegetation index), as shown in the following Table 3.

Table 3 Vegetation index image number of original information

\begin{tabular}{ccc}
\hline$V I$ & $V I_{\text {soil }}$ & $V I_{\text {veg }}$ \\
\hline$N D V I$ & 0.033877 & 0.666737 \\
$G N D V I$ & 0.107601 & 0.582231 \\
$E V I$ & 0.021377 & 0.448939 \\
$D V I$ & 0.017223 & 0.249841 \\
$S A V I$ & 0.024086 & 0.423150 \\
$R V I$ & 1.031326 & 5.016646 \\
OSAVI & 0.026761 & 0.463123 \\
\hline
\end{tabular}

According to the above vegetation coverage model combined with the $V I_{\text {soil }}$ and $V I_{\text {veg }}$ information of each vegetation index in Table 3, the vegetation coverage map of summer maize in the Jointing period is calculated. Because the difference in vegetation coverage images extracted by each model is small, only EVI vegetation is displayed. The vegetation coverage map obtained from the coverage extraction model is shown in Figure 4.

Calculate the vegetation coverage map obtained from the calculation of each model to obtain the average vegetation coverage of the entire test area. At the same time, perform statistics on the SVM supervision classification map to obtain the true value of the vegetation coverage to evaluate the accuracy of the vegetation coverage extracted by each model. The results are 
shown in Table 4 below.

It can be seen from Table 4 that the vegetation coverage model constructed based on the pixel dichotomy using the vegetation index RVI has the worst extraction effect on vegetation coverage, with an extraction error of $26.72 \%$. The NDVI vegetation coverage model has a better extraction effect on vegetation coverage. The error is $12.62 \%$, and the RVI coverage extraction model has the best extraction effect on vegetation coverage, with an extraction error of $11.76 \%$.

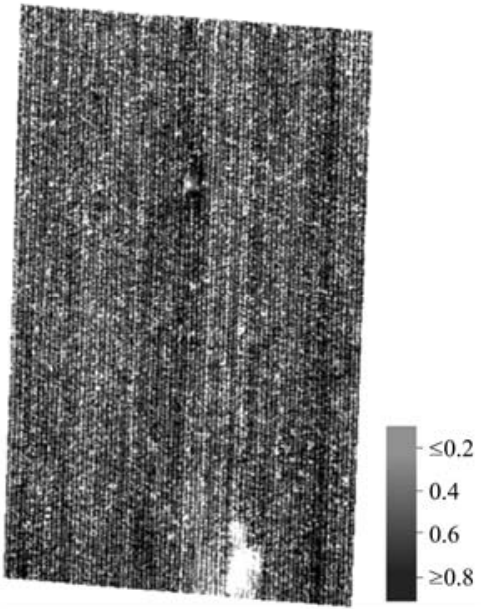

Figure 4 EVI-Vegetation coverage map
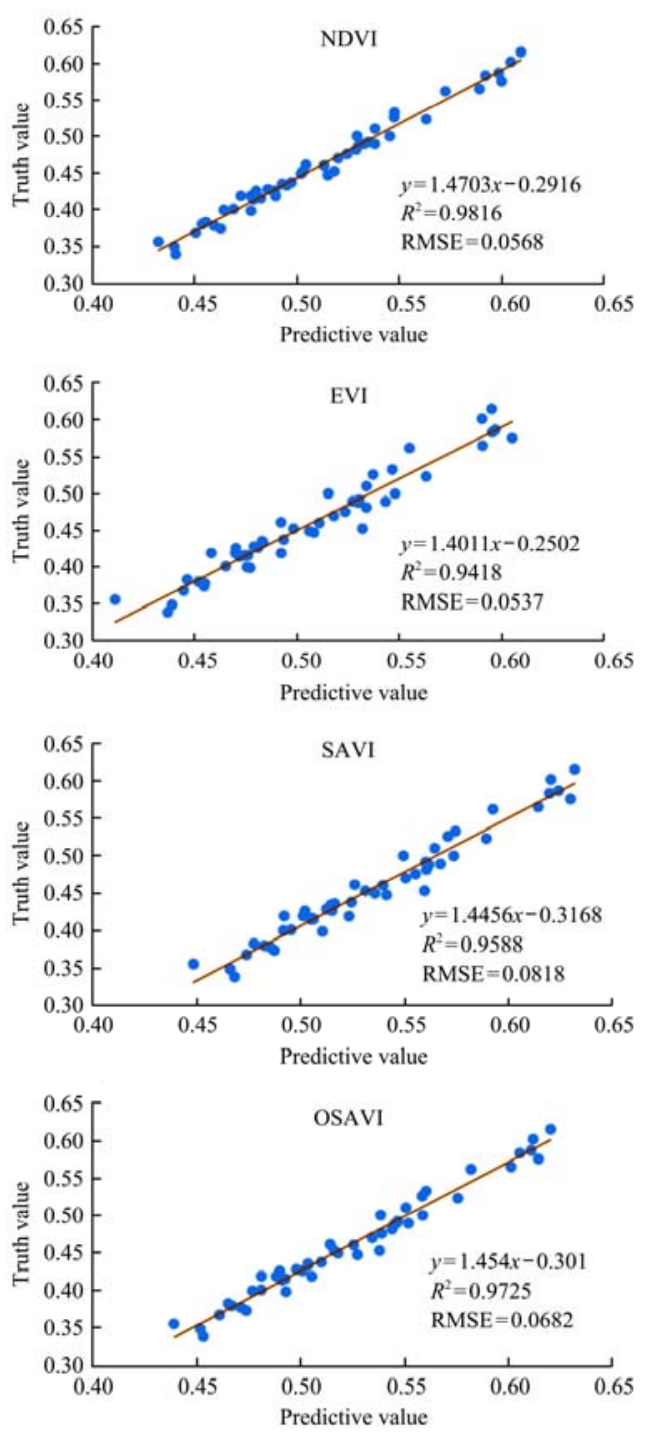

Table 4 Precision evaluation

\begin{tabular}{|c|c|c|c|c|}
\hline \multirow{2}{*}{$\begin{array}{l}\text { Vegetation } \\
\text { index model }\end{array}$} & \multicolumn{3}{|c|}{ Vegetation coverage } & \multirow{2}{*}{$\begin{array}{c}\text { Extraction } \\
\text { error }\end{array}$} \\
\hline & $\begin{array}{c}\text { Pixel } \\
\text { dichotomy }\end{array}$ & $\begin{array}{l}\text { Supervised } \\
\text { classification }\end{array}$ & Difference & \\
\hline$F V C_{N D V I}$ & 0.499704 & 0.443692 & 0.056012 & 12.62 \\
\hline$F V C_{G N D V I}$ & 0.519437 & 0.443692 & 0.075745 & 17.07 \\
\hline$F V C_{E V I}$ & 0.495880 & 0.443692 & 0.052188 & 11.76 \\
\hline$F V C_{D V I}$ & 0.536505 & 0.443692 & 0.092813 & 20.92 \\
\hline$F V C_{S A V I}$ & 0.526461 & 0.443692 & 0.082769 & 18.65 \\
\hline$F V C_{R V I}$ & 0.325151 & 0.443692 & 0.118541 & 26.72 \\
\hline$F V C_{O S A V I}$ & 0.512110 & 0.443692 & 0.068418 & 15.42 \\
\hline
\end{tabular}

In order to further verify the extraction accuracy of the vegetation coverage model, this paper uniformly selects 50 100 Pixel $\times 100$ Pixel cells in the test area ${ }^{[30-35]}$, and calculates the vegetation coverage of the 50 selected cells in the vegetation coverage map obtained from each vegetation coverage model. Take the average value of the vegetation coverage in each cell as the predicted value of the vegetation coverage of the cell, and extract the average value of the vegetation coverage in the 50 cells corresponding to the SVM supervision classification map as the true value, and linearize the predicted value and the true value respectively Fit, calculate the coefficient of determination $\left(R^{2}\right)$ and root mean square error RMSE of each scatter plot, and the linear fitting result is shown in Figure 5.
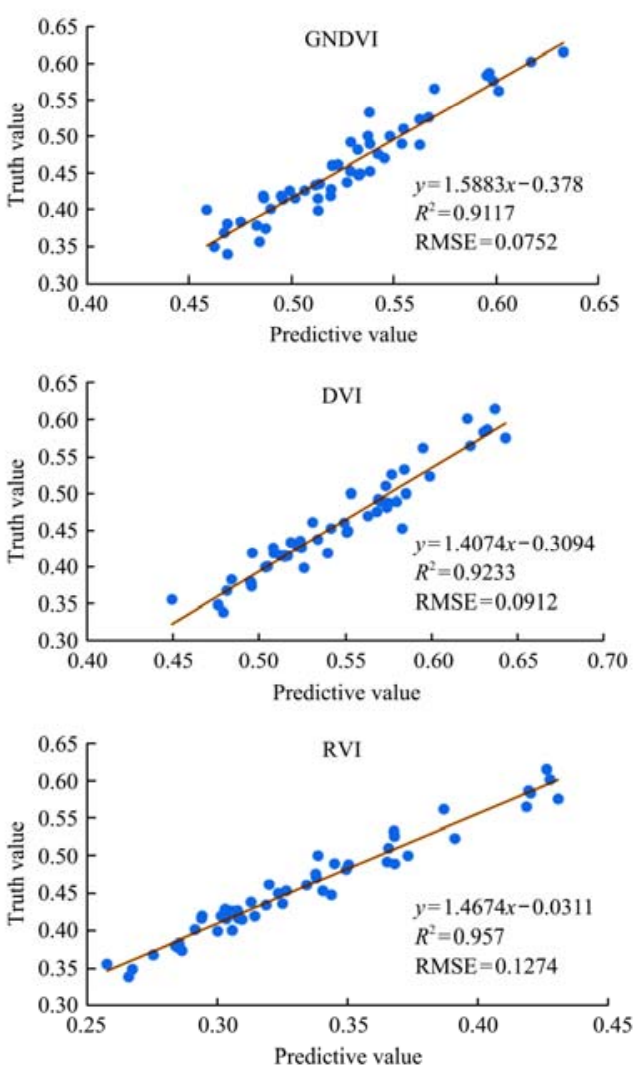

Figure 5 Linear fitting results of vegetation coverage extraction model 
Table 5 The error analysis

\begin{tabular}{ccc}
\hline$V I$ & $R^{2}$ & $R M S E$ \\
\hline$N D V I$ & 0.9816 & 0.0568 \\
$G N D V I$ & 0.9117 & 0.0752 \\
$E V I$ & 0.9418 & 0.0537 \\
$D V I$ & 0.9233 & 0.0912 \\
SAVI & 0.9588 & 0.0818 \\
$R V I$ & 0.9570 & 0.1274 \\
OSAVI & 0.9725 & 0.0682 \\
\hline
\end{tabular}

From Figure 5 and Table 5, it can be seen that the extraction effect of GNDVI and DVI in the 7 plant coverage extraction model is poor, the coefficient of determination $\left(R^{2}\right)$ is 0.9117 and 0.9233 , and the RMSE is 0.0752 and 0.0912 , respectively; the other vegetation indices in the comparison table Corresponding parameters of the coverage extraction model, the $R^{2}$ of GNDVI and DVI are small, the fitting effect of the true value and the predicted value is poor, the RMSE value is large, and the true value and the predicted value are more discrete.

The overall extraction effect of NDVI and EVI is better, the coefficients of determination $\left(R^{2}\right)$ of the two are 0.9816 and 0.9418 , respectively, and the RMSE are 0.0568 and 0.0537 , respectively. The true value is close to the predicted value, and the fitting effect is good. The results are compared with those obtained in Table 4 . The accuracy results of the whole image verification are consistent.

Combining the two accuracy verification results, it is concluded that the degree coverage extraction model constructed by using the EVI vegetation index based on the pixel dichotomy has the best effect and high accuracy.

In order to guide agricultural production and facilitate field management operations, the vegetation coverage of summer maize is further classified. According to the method proposed by Chen Hongbing ${ }^{[36]}$, this paper selects the EVI vegetation coverage model to obtain the vegetation coverage grade distribution of summer maize in the jointing period. The figure, as shown in the figure, this article divides a total of 10 levels, and the statistical results of the coverage of each level are shown in Table 6 and Figure 6.

Table 6 Results of distribution of vegetation coverage grades

\begin{tabular}{ccc}
\hline Vegetation coverage & Like the original number & Percentage/\% \\
\hline $0 \sim 0.1$ & 377941 & 14.780 \\
$0.1 \sim 0.2$ & 200353 & 7.835 \\
$0.2 \sim 0.3$ & 285005 & 11.146 \\
$0.3 \sim 0.4$ & 302694 & 11.838 \\
$0.4 \sim 0.5$ & 284553 & 11.128 \\
$0.5 \sim 0.6$ & 262911 & 10.282 \\
$0.6 \sim 0.7$ & 251540 & 9.837 \\
$0.7 \sim 0.8$ & 234134 & 9.156 \\
$0.8 \sim 0.9$ & 190975 & 7.469 \\
$0.9 \sim 1$ & 166938 & 6.529 \\
Total & 2557044 & 100 \\
\hline
\end{tabular}

\section{Conclusions}

The vegetation index vegetation coverage extraction model based on UAV multi-spectral images can be used to efficiently and accurately extract the vegetation coverage of summer maize:

(1) A vegetation coverage extraction model developed by using the seven common vegetation indices of multispectral combined with the principle of pixel dichotomy can be applied to effectively extract the vegetation coverage information of summer maize in the jointing period.

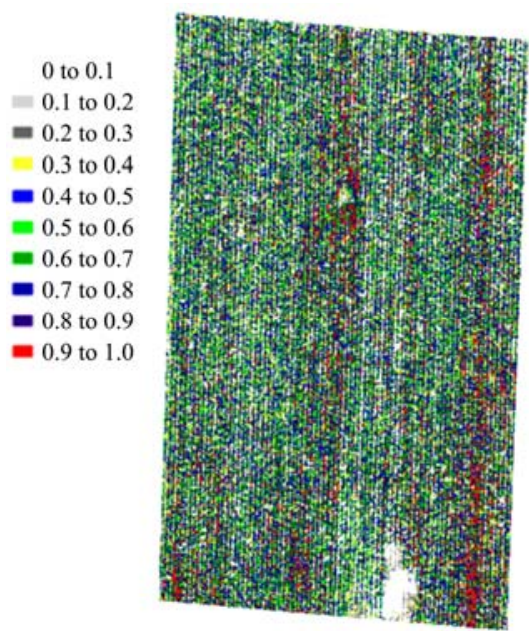

Figure 6 Distribution map of vegetation coverage grade of summer maize

(2) Based on the pixel dichotomy, the vegetation coverage extraction model constructed by the 7 planting index was used to extract the vegetation coverage of summer maize in the Jointing period. Among them, the coverage extraction model constructed by the EVI vegetation index had the highest accuracy and the extraction error $\left(E_{F}\right)$ Is $11.76 \%, R_{2}$ is 0.9418 , RMSE: 0.0537 .

(3) Use the EVI vegetation coverage extraction model to generate the vegetation coverage rating map of the summer maize in the Jointing period to provide technical support for the realization of precision agriculture and the guidance of field management.

\section{Acknowledgments}

This study was supported by Top Talents Program for One Case One Discussion of Shandong Province and the Development Special Funds on Science and Academy of Ecological Unmanned Farm.

\section{[References]}

[1] Weng L Y. Analysis of the current situation of corn production in China and its development countermeasures. China Food and Nutrition, 2010(1) 22-25. (in Chinese)

[2] Lan Y B. Current status and future prospects of precision agricultural aviation technology. Agricultural Engineering Technology, 2017, 37(30): 27-30. doi: 10.16815/j.cnki.11-5436/s.2017.30.003. (in Chinese)

[3] He D J, He Y, Li M Z, Hong T S, Wang C H, Song S, Liu Y G. Research progress on information-related scientific issues in precision agriculture. National Science Foundation of China, 2011, 25(1): 10-16. doi: 10.16262/j.cnki.1000 -8217.2011.01.006. (in Chinese )

[4] Jia K, Yao Y J, Wei X Q, et al. Research progress on remote sensing estimation of vegetation coverage. Advances in Earth Science, 2013, 28(7): 774-782. (in Chinese)

[5] Niu Y X, Zhang L Y, Han W T. Research on the extraction method of cotton coverage based on Lab color space. Transactions of the Chinese Society of Agricultural Machinery, 2018, 49(10): 240-249. (in Chinese)

[6] Li M M, Wu B F, Yan C Z, et al. Remote sensing estimation of vegetation coverage in the upper reaches of Miyun Reservoir. Resources Science, 2004, 26(4): 153-159. (in Chinese)

[7] Zhao J, Yang H B, Lan Y B, et al. Extraction method of summer corn vegetation coverage based on UAV visible light image. Transactions of the Chinese Society of Agricultural Machinery, 2019, 50(5): 232-240. (in Chinese)

[8] Niu Y X, Zhang L Y, Han W T, et al. Winter wheat coverage extraction method based on UAV remote sensing and vegetation index. Transactions of the Chinese Society of Agricultural Machinery, 2018, 49(4): 212-221. (in Chinese)

[9] Wang X Q, Wang M M, Wang S Q, et al. Vegetation information extraction based on UAV remote sensing in the visible light band. Transactions of the Chinese Society of Agricultural Engineering, 2015, 
31(5): 152-159. (in Chinese)

[10] Ciliies R R. A method to make use of thermal infrared temperature and NDVI measurements to infer surface soil water content and fractional vegetation cover. Remote Sensing Reviews, 1994, 9(1): 161-173.

[11] Bausch W C. Soil background efects on reflectance-hased crop coeficients for corn. Renote Sensing of Envionmen, 1993, 46(2): 213-222.

[12] Zhang X, Liao C, Li J, et al. Fractional veetation cover estimation in arid and semi-urid environmens using $\mathrm{HJ}$ - satellite hyperspectral data International Journal of Applied Earth Ohservation and Geoinformation, 2013, 21: 506-512. doi: 10.1016/j.jag.2012.07.003. (in Chinese)

[13] Jia K, Li Y W, Liang S, et al. Fractional vegetation eover estimation based on soil and vegetation lines in a corn-lominatedl area. Geocarto International, 2016, 32(5): 531-540. doi: 10.1080/10106049.2016.1161075. (in Chinese)

[14] Dai J G, Zhang G S, Guo P, et al. Classification method of main crops in northern Xinjiang based on UAV remote sensing visible light images Ding. Transactions of the Chinese Society of Agricultural Engineering, 2018, 34 (18): 122-129. (in Chinese)

[15] Tong S Q, Bao Y H, Zhang Q F, et al. Analysis of spatiotemporal changes of vegetation coverage in Inner Mongolia based on pixel dichotomy and intensity analysis method. Acta Eco-Environmental Sciences, 2016, 25(5): 737-743. doi: 10.16258/j.cnki.1674-5906.2016.05 002. (in Chinese)

[16] Liu C, Yang G J, Li Z H, Tang F Q, Wang J W, Zhang C L, Zhang L Y. Winter wheat biomass estimation fusion of UAV spectral information and texture information. Chinese Agricultural Sciences, 2018, 51(16): 3060-3073. (in Chinese)

[17] Liu Z Y, Huang M F, Wu X H, et al. Hyperspectral remote sensing estimation models of natural grassland vegetation coverage. Chinese Journal of Applied Ecology, 2006, 17(6): 997-1002. (in Chinese)

[18] Han W T, Guo C C, Zhang L Y, et al. Classification method of land use and cover in irrigation area based on UAV remote sensing. Transactions of the Chinese Society of Agricultural Machinery, 2016, 47(11). (in Chinese)

[19] Zhang Y F. Estimation of Winter Wheat Vegetation Coverage Based on Multi-source Remote Sensing Data. Nanchang: East China University of Technology, 2016. (in Chinese)

[20] Ts. P R, et al. Relationships between percent vegetation cover and vegetation indices. International Journal of Remote Sensing, 1998, 19(18) 3519-3535. doi: 10.1080/ 014311698213795.

[21] Liu Y K, Mu X H, Wang H X, et al. A novel method for extracting green fractional vegetation cover from dital images. Jouralof Vegetation Science, 2012, 23(3): 406-418. doi: 10.1111/j.1654-1103.2011.01373.x.

[22] Bradley C R. The influence of canopy green vegetation fraction on spectral measurements over native tallgrass prairie. Remote Sensing of Environment, 2002, 81(1): 129-135. doi: 10.1016/S0034-4257(01) 00339-X.
[23] Gregory S O, Kenneth D, Clarke M M. Lewis. Comparison of methods for estimation of absolute vegetation and soil fractional cover using MODIS normalized BRDF-adjusted reflectance data. Remote Sensing of Environment, 2013, 130: 266-279. doi: 10.1016/j.rse.2012.11.021.

[24] Ciliies R R. A method to make use of thermal infrared temperature and NDVI measurements to infer surface soil water content and fractional vegetation cover. Remote Sensing Reviews, 1994, 9(1): 161-173.

[25] Bi Z M, et al. Derivation of wild vegetation cover density in semi-arid regions: ERS2/SAR evaluation. Int J Remote Sens, 2003, 24(6): 1335-1352. doi: 10.1080/01431160210146668.

[26] Li C J, Wang J H, Liu L Y, et al. Automatic extraction of wheat coverage based on digital photo features. Journal of Zhejiang University: Agriculture and Life Sciences Edition, 2004, 30(6): 64-70. (in Chinese)

[27] Li D K, Fan J Z, Wang J. Variation characteristics and causes of vegetation coverage in Shaanxi Province. Journal of Applied Ecology, 2010, 21(11): 2896-2903. (in Chinese)

[28] Li B, Liu R Y, Liu S H, et al. Monitoring of winter wheat coverage changes based on low-altitude UAV remote sensing. Transactions of the Chinese Society of Agricultural Engineering, 2012, 28(13): 160-165. (in Chinese)

[29] Wang P, Luo X W, Zhou Z Y, et al. Overview of key technologies for remote sensing information acquisition based on micro-unmanned aerial vehicles. Transactions of the Chinese Society of Agricultural Engineering, 2014, 30(18): 1-12. (in Chinese)

[30] Wang T T, An Y G, Li J T, Xiao L L, et al. Seed coating provides rice seeds for aerial seeding with reduced phenamacril loss and a prolonged rice seedling protection, 2021; 4(1): 70-74. (in Chinese)

[31] Yang D J, Zhao J, Lan Y B, et al. Research on farmland crop classification based on UAV multispectral remote sensing images. 2021; 4(1): 29-35. doi: 10.33440/j. ijpaa.20210401.

[32] Yang H B, Hu X, Zhao J, Hu Y H. Feature extraction of cotton plant height based on DSM difference method. 2021; 4(1): 59-69. doi: 10.33440/j.ijpaa.20210401.151.

[33] Xu P F, Wang H C, Yang S H, Zheng Y J. Detection of crop heights by UAVs based on the Adaptive Kalman Filter. Int J Precis Agric Aviat, 2021; 4(1): 52-58. doi: 10.33440/j. ijpaa.20210401.166.

[34] Meng Y H, Wang M M, Wang Z G, Hu H Y, Ma Y. Surface tension and spreading coefficient of single-and mix-pesticide solutions with aerial spraying organosilicone adjuvant. Int J Precis Agric Aviat, 2021; 4(1): 6-13. doi: 10.33440/j.ijpaa.20210401.159.

[35] Li S D, Zhao W, Ji J, Hu C Q, Hu X Q. Analysis of flow resistance property for valve-less piezoelectric pump with hemisphere-segment bluff-body. Int J Precis Agric Aviat, 2021; 4(1): 14-21. doi: 10.33440/ j.ijpaa.20210401.149.

[36] Chen H B, Huang B B, Peng D L. Comparison of vegetation coverage models based on mixed pixel decomposition. Journal of Northwest Forestry University, 2018, 33(3): 203-207. (in Chinese) 